\title{
An unusual case of dysphonia and dysphagia
}

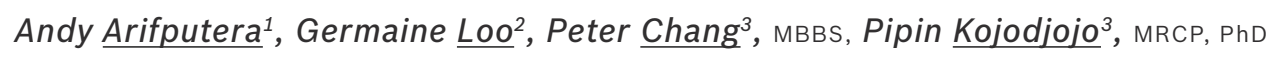

\begin{abstract}
A 68-year-old man presented with a six-month history of painless dysphagia, malnutrition, anorexia and vocal hoarseness. Signs of severe mitral regurgitation and preserved left atrial dimensions were discovered on transthoracic echocardiography. However, electrocardiography and chest radiography were strongly suggestive of left atrial enlargement. Further investigations confirmed extrinsic compression of the oesophagus, which caused the dysphagia. Computed tomography of the throrax revealed a giant left atrium that was not appreciated on echocardiography. Hoarseness was found to be caused by right recurrent laryngeal nerve palsy. Ortner's syndrome, which describes the occurrence of vocal hoarseness due to a cardiopulmonary disease that results in the compression of the left recurrent laryngeal nerve, is usually associated with severe mitral stenosis. Herein, we report an unusual case of Ortner's syndrome caused by a giant left atrium, which resulted from severe mitral regurgitation, causing extrinsic oesophageal compression and right recurrent laryngeal nerve palsy. Physicians should remain cognisant of cardiovascular disorders as uncommon causes of painless dysphagia or vocal hoarseness.
\end{abstract}

Keywords: cardiovocal syndrome, dysphagia, left atrial enlargement, mitral regurgitation, right recurrent laryngeal nerve palsy

\section{INTRODUCTION}

Ortner's syndrome, more commonly known as cardiovocal syndrome, is a clinical entity characterised by hoarseness that is attributable to a cardiovascular disease resulting in left recurrent laryngeal nerve palsy. Ortner, in 1897, first described this syndrome in a patient with mitral stenosis and left atrial enlargement. ${ }^{(1)}$ Since its first description, various underlying cardiopulmonary causes resulting in left recurrent laryngeal nerve palsy have been well documented. ${ }^{(2)}$ Herein, we report the case of a patient who presented with atypical Ortner's syndrome and painless dysphagia.

\section{CASE REPORT}

A 68-year-old Chinese man was admitted for painless dysphagia associated with a six-month history of nausea, malnutrition, weight loss and hoarseness. Right vocal cord palsy was detected during nasoendoscopy. Physical examination revealed that the patient was severely underweight with a body mass index of $14 \mathrm{~kg} / \mathrm{m}^{2}$. His blood pressure was $115 / 70 \mathrm{mmHg}$, and his heart rate was $70 \mathrm{bpm}$. Both the first and second heart sounds were audible, with pansystolic murmur detected the apical region. The patient's jugular venous pressure was not elevated, but bibasal crackles were present. Chest radiography demonstrated overall cardiomegaly and signs of left atrial dilatation, as evidenced by an additional right heart border and a prominent bulge in the left heart border (Fig. 1). The patient's resting 12-lead electrocardiography (ECG) showed sinus rhythm (Fig. 2). The $\mathrm{P}$ wave was notched and prolonged, with a duration of more than $110 \mathrm{~ms}$. The biphasic $\mathrm{P}$ wave was present in leads $\mathrm{V} 1$ and $\mathrm{V} 2$, with the duration of the negative phase greater than

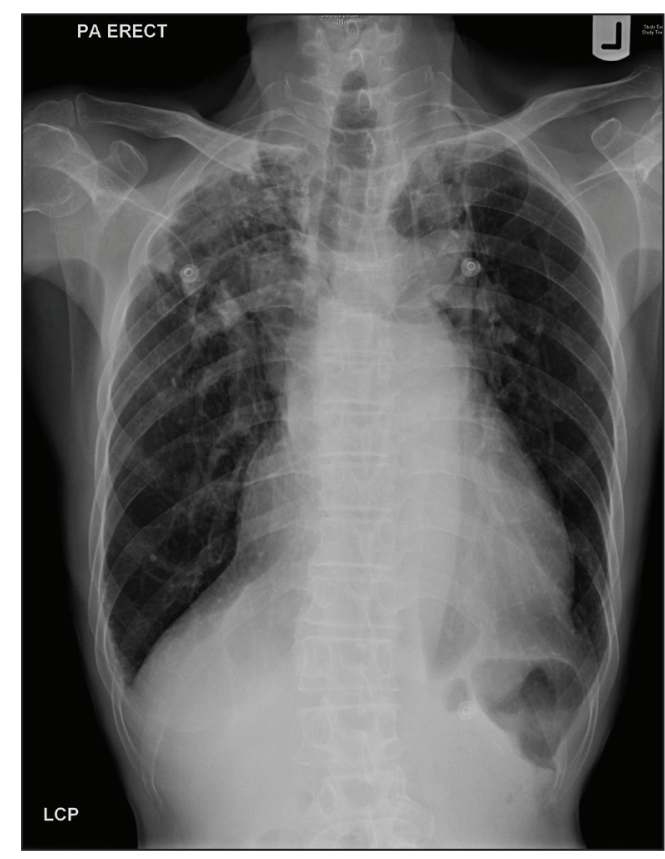

Fig. 1 Chest radiograph shows features of cardiomegaly and left atrial dilatation. Note the extra right heart border and prominent bulge along the left heart border, both of which are features of left atrial enlargement.

$40 \mathrm{~ms}$. These ECG features are also highly suggestive of left atrial enlargement.

Barium swallow revealed an external indentation of the anterior oesophagus at the level of the aortic arch, with resultant dilatation of the proximal oesophagus (Fig. 3). This was attributed to either cardiomegaly or an unfolded aorta. Transthoracic echocardiogram (TTE) showed a preserved left atrium, with dimensions of $38 \mathrm{~mm}$ in the parasternal long axis

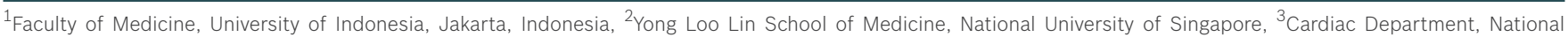
University Heart Centre, Singapore

Correspondence: Dr Pipin Kojodjojo, Consultant Cardiologist, Cardiac Department, National University Heart Centre, 1E Kent Ridge Road, NUHS, NUHS Tower Block, Level 9, Singapore 119228. pipin_kojodjojo@nuhs.edu.sg
} 


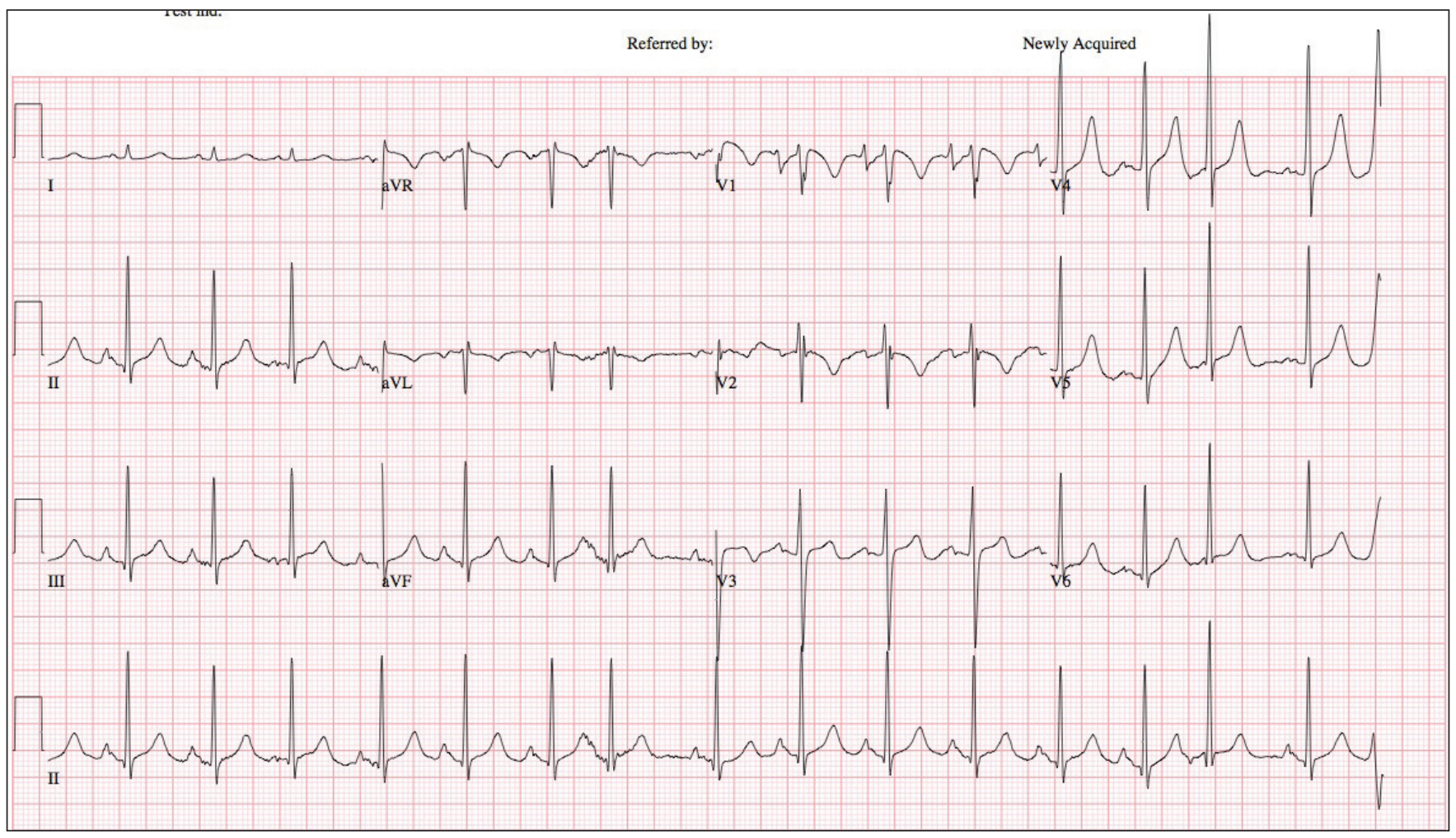

Fig. 2 ECG shows features of left atrial enlargement.

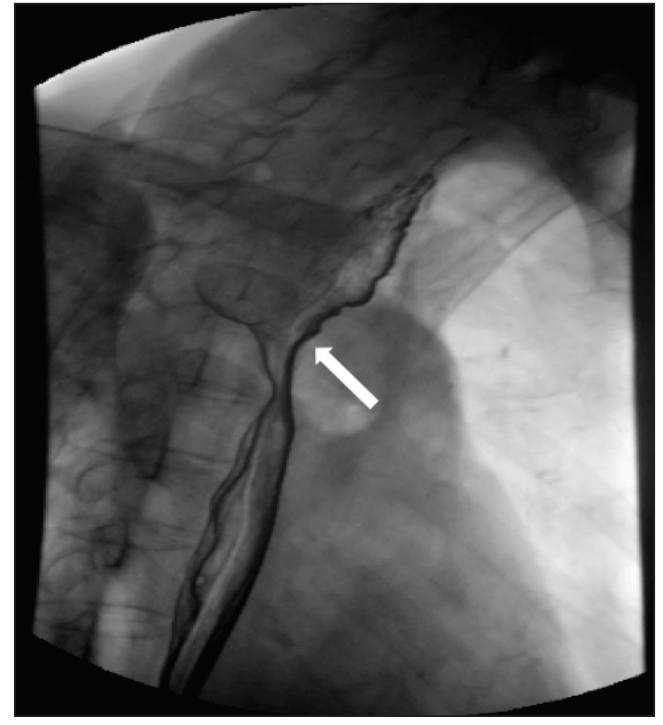

Fig. 3 Barium swallow image shows an external indentation (arrow) of the oesophagus.

view and $42 \mathrm{~mm} \times 45 \mathrm{~mm}$ in the apical four-chamber view, and normal left ventricular systolic function (Fig. 4). The left ventricular end diastolic dimension was $53 \mathrm{~mm}$. These left atrial measurements were made at the end of ventricular systole, when the left atrium was at its largest dimension. Using the three measured left atrial dimensions, the left atrial volume was calculated using the ellipsoid formula. The indexed left atrial volume was $17 \mathrm{~mL} / \mathrm{m}^{2}$ (adjusted for body surface area; left atrial dilatation was defined as an indexed left atrial volume of $>29 \mathrm{~mL} / \mathrm{m}^{2}$ ), which was not consistent with our clinical suspicion of left atrial enlargement. There was severe mitral valve regurgitation due to a flail posterior mitral valve leaflet.
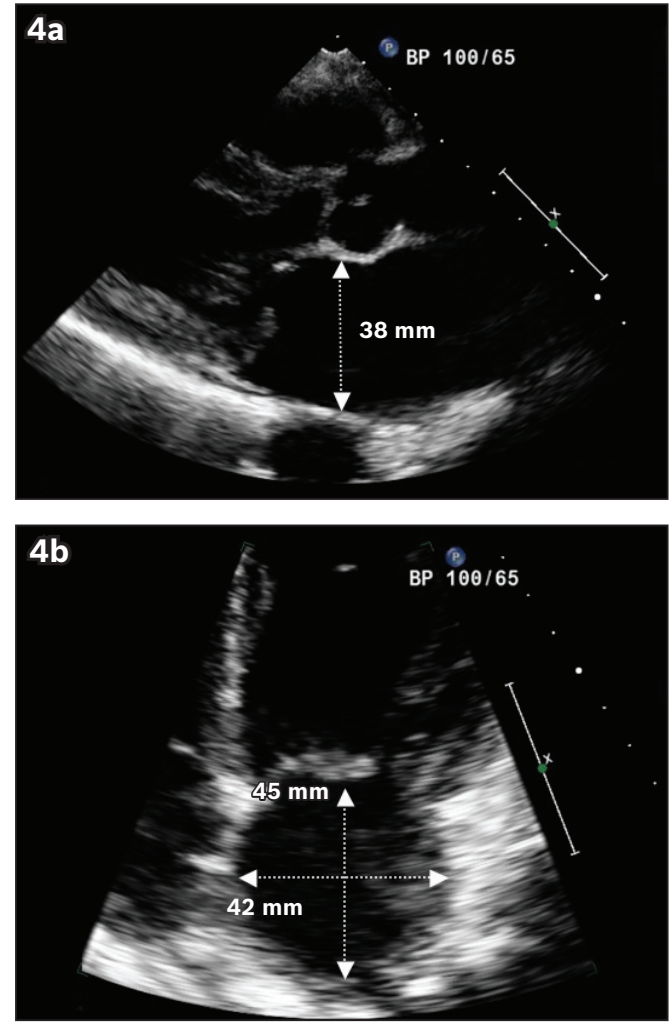

Fig. 4 Transthoracic echocardiogram shows the left atrial dimensions, measured at the end of ventricular systole, in the (a) parasternal long axis and (b) apical four-chamber view.

The pulmonary artery was not dilated, with pulmonary arterial systolic pressure estimated to be $30 \mathrm{mmHg}$.

Computed tomography (CT) of the thorax was performed to exclude noncardiac causes of extrinsic oesophageal compression, and it confirmed a grossly dilated left atrium 


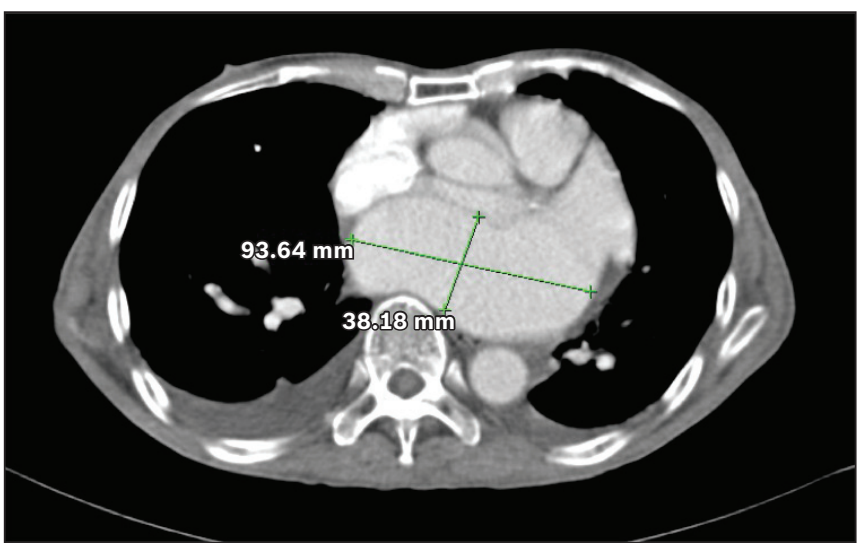

Fig. 5 CT shows marked dilatation of the left atrium.

(measuring $94 \mathrm{~mm} \times 38 \mathrm{~mm}$ ) that was severely underestimated by TTE. The grossly dilated left atrium was found indenting the oesophagus (Fig. 5). The patient was provided with thickened feeds and calorific supplementation, and referred for expedient mitral valve repair.

\section{DISCUSSION}

Ortner's syndrome is characterised by hoarseness due to a myriad of cardiovascular diseases that cause the compression of the left recurrent laryngeal nerve along its anatomical course, resulting in left vocal cord palsy. In the literature, Ortner's syndrome is most commonly associated with severe mitral stenosis. (2) Other causes include primary pulmonary hypertension, mitral valve disease, left atrial myxoma, recurrent pulmonary artery embolism, aortic aneurysm and congenital heart diseases. ${ }^{(2)}$ Ortner first attributed the cause of left recurrent laryngeal nerve compression to an enlarged left atrium due to mitral stenosis. ${ }^{(1)}$

The present case was unusual for several reasons. Firstly, while Ortner's syndrome is usually caused by disruption of the left recurrent laryngeal nerve, it was the right recurrent laryngeal nerve that was affected in our patient. Since the right recurrent laryngeal nerve is the terminal branch of the vagus nerve, it crosses the first part of the subclavian artery before hooking around the subclavian artery to travel between the trachea and oesophagus, in its path to the larynx, where it supplies motor and sensory innervation. A grossly dilated left atrium compresses the oesophagus, which in turn compresses the right recurrent laryngeal nerve between the oesophagus and trachea, resulting in right vocal cord palsy. To the best of our knowledge, such a presentation has not been reported previously.

Secondly, despite the presence of severe mitral regurgitation, our patient presented with noncardiovascular symptoms of hoarseness and dysphagia, which led to malnutrition and severe weight loss. These symptoms were caused by the extrinsic compression of the oesophagus by the giant left atrium, which arose from severe mitral regurgitation. Cardiovascular dysphagia, another complication of left atrial enlargement, was first reported by Morgan and Mourant in 1980. ${ }^{(3)}$ However, the condition is rarely diagnosed and its occurrence, together with Ortner's syndrome, has not been previously reported. Lastly, our patient's ECG and chest radiography showed prominent features of left atrial enlargement that was unappreciated on TTE, but confirmed on CT of the thorax.

Left atrial enlargement is defined electrocardiographically as one of the following: (a) negative phase of $\mathrm{P}$ wave in lead V1 lasting > $40 \mathrm{~ms}$; (b) negative phase in V1 > $1 \mathrm{~mm}$ deep; (c) P-terminal force $>40 \mathrm{mV} / \mathrm{ms}$; (d) notched $\mathrm{P}$ wave with interpeak interval > $40 \mathrm{~ms}$; and (e) $\mathrm{P}$ wave duration > $110 \mathrm{~ms}$. Of the aforementioned criteria, a negative phase duration of $\mathrm{P}$ wave in $\mathrm{V} 1$ that is $>40 \mathrm{~ms}$ is the most sensitive electrocardiographic feature, while notched $P$ wave is the most specific feature. ${ }^{(4)}$ The ECG presented in Fig. 1 fulfilled all the diagnostic criteria that are considered trademarks of interatrial conduction delay.

A diagnosis of left atrium enlargement is usually confirmed on TTE. However, in our patient, the left atrium size was severely underestimated by TTE. While easily accessible and relatively inexpensive, TTE is limited to two-dimensional imaging of the cardiac chambers. It is also subject to operator-dependent variability in image acquisition, as well as the physical restrictions of acoustic windows. Other more expensive modalities such as three-dimensional echocardiography, CT and magnetic resonance imaging can provide a more thorough measurement of the left atrial size. If ECG, chest radiography and clinical findings are consistent with left atrial enlargement, an alternative imaging modality could be considered if TTE is not diagnostic.

In conclusion, the present study highlights the importance of including cardiovascular disease in the differential diagnoses of patients presenting with hoarseness and/or painless dysphagia. Although TTE is usually diagnostic for left atrial enlargement, clinicians should remain cognisant of the limitations of TTE, which were illustrated in the present case.

\section{ACKNOWLEDGEMENT}

We thank Prof Chia Boon Lock for his advice and help during the writing of this article.

\section{REFERENCES}

1. Ortner N. Recurrent laryngeal nerve paralysis due to mitral valve stenosis. Wien Klin Wochenschr 1897; 10:753-5.

2. Mulpuru SK, Vasavada BC, Punukollu GK, Patel AG. Cardiovocal syndrome: a systematic review. Heart Lung Circ 2008; 17:1-4.

3. Morgan AA, Mourant AJ. Left vocal chord paralysis and dysphagia in mitral valve disease. Br Heart J 1980; 43:470-3.

4. Waggoner AD, Adyanthaya AV, Quinones MA, Alexander JK. Left atrial enlargement. Echocardiographic assessment of electrocardiographic criteria. Circulation 1976; 54:553-7. 